\title{
The Vulnerabilities of Orphaned Children Participating in Research: A Critical Review and Factors for Consideration For Participation in Biomedical and Behavioral Research
}

Rachel T. Thompson, Eric M. Meslin, and Paula K. A. Braitstein

Indiana University (USA)

Winstone M. NyANDiKo AND

Samuel O. Ayaya

Moi University (Kenya)

RACHEL C. VREEMAN

Indiana University (USA)

ABSTRACT: ORPHANS ARE A SUBPOPULATION WITH a unique set of additional vulnerabilities. Increasing focus on children's rights, pediatric global health, and pediatric research makes it imperative to recognize and address unique vulnerabilities of orphaned children. This paper describes the unique vulnerabilities of the orphaned pediatric population and offers a structured set of factors that require consideration when including orphans in biomedical research. Pediatric orphans are particularly vulnerable due to decreased economic resources, psychosocial instability, increased risk of abuse, and delayed/decreased access to healthcare. These vulnerabilities are significant. By carefully considering each issue in a population in a culturally specific and study-specific manner, researchers can make valuable contributions to the overall health and well-being of this uniquely vulnerable population.

KEY WORDS: orphan, vulnerability, research, HIV, Kenya

Received: June 21, 2012; Revised: September 12, 2012

$\mathbf{P}$ OPULATIONS CAN BE DEFINED AS "VULNERABLE" using various parameters (Levine et al., 2004). Participants may be vulnerable due to socioeconomic status, age, gender, or simply the timing of the particular study as may occur in times of catastrophic illness, natural disasters, and political upheaval. Most regulatory and oversight documents for research involving human subjects obligate researchers to provide additional protections to vulnerable populations. For example, in the United States additional protections are specifically described for prisoners, pregnant women and fetuses, and children (US Department of Health and Human Services, n.d.). The ethical and legal obligation to provide additional protections to vulnerable subjects is derived from recognized principles of bioethics, including those three initially described in the Belmont Report: respect for persons, justice, and beneficence (US Department of Health and Human Services, 1979). Refinements to these principles, for example by Beauchamp and Childress, have enjoyed support, and are also reflected in major international guidance documents including the Declaration of Helsinki (Beauchamp, 2009). The common ethical commitment found in all protections of vulnerable research subjects is to protect those incapable of protecting themselves against harm or exploitation.

But context and geography matter. Despite the emphasis on these protections, challenges arise as researchers begin to include increasingly vulnerable populations, such as African children, in their studies. Even though ethical principles may enjoy support, their local interpretation matters. Basic definitions of who comprises a population such as "orphaned children" may be a source of contention, while other definitions such as children as a vulnerable population may not be universally accepted.

Kipnis (2003) has described seven specific vulnerabilities of the pediatric population, including the possible undervaluing of their rights and interests in society and the subjugation of their authority to others (Figure 1). The ethical obligation to protect vulnerable pediatric research participants from harm is met largely through two mechanisms: (1) the additional protections that institutional review boards may impose on researchers, and (2) the involvement of legally authorized representatives-most often a parent or guardian to act in the participant's best interests. But what should happen when parents or guardians do not exist? Orphaned children have no such advocates, and therefore no one to act in their best interests.

Journal of Empirical Research on Human Research Ethics, VOL. 7, No. 4, PP. 56-66. PRINT ISSN 1556-2646, ONLINE ISSN 1556-2654. (C) 2012 By JOAN SIEBER. ALL RIGHTS RESERVED. PLEASE DiRECT ALl REQUESTS For PERMisSions to PHOTOCOPY OR REPRODUCE ARTICLE CONTENT THROUGH THE UNIVERSITY OF CALIFORNIA PRESS'S RIGHTS AND PERMISSIONS WEBSITE, HTTP://WWW.UCPRESSJOURNALS.COM/REPRINTINFO.ASP. DOI: $10.1525 /$ jer.2012.7.4.56 


\section{Seven Vulnerabilities of Pediatric Populations}

(1) Commonly lack the capacity to make mature decisions

(2) Subject to authority of others

(3) They may be deferential in ways that mask underlying dissent

(4) Their rights and interests may be socially undervalued

(5) They may have acute medical conditions requiring immediate decisions not consistent with informed consent

(6) They may have serious medical conditions that cannot be effectively treated

(7) They may lack important socially distributed goods

FIG. 1. Kipnis's Seven Vulnerabilities.

It is unclear how to ensure adequate protection for this vulnerable population, and raises the question of whether orphans should be included in health research at all. The necessity requirement dictates that vulnerable individuals should be included only if one could not do the research using less vulnerable populations. Additionally, the subject requirement demands that the research addresses a question that is unique to the vulnerable population. Following these requirements might result in the exclusion of children from research, to the detriment of the pediatric population. Although this may seem protective, there are many important reasons to include children in research. For example, across the developmental spectrum from infancy through adolescence, children have different physiologic and metabolic profiles that influence the effects of a medication, making it imperative to evaluate medications in children and not just in adults. Similarly, the orphan population should not be summarily dismissed from biomedical research. Orphans face particular physical, social, and emotional challenges, for which the effects and solutions may only be illuminated if research is conducted in this particular population. Both the necessity and the subject requirements should be carefully considered and applied to each new protocol involving orphans. As orphans are subject to a substantial share of health burdens, researchers may also find that the principle of justice directs us to answer important questions to improve the health and care of the 132 million orphans in subSaharan Africa, Asia, Latin America, and the Caribbean (UNICEF, 2009).

\section{A Theoretical Case Study in the Vulnerabilities of Orphans in Research Ethics}

Consider a theoretical case of a proposed HIV vaccine trial in rural Kenya. Consideration of this case will serve as a springboard to conduct a critical review of the participation of orphans in health research, and then yield a series of distinct factors that must be considered for the participation of orphans in biomedical and behavioral research.
PREVALENCE OF ORPHANHOOD DEMANDS CONSIDERATION FOR HEALTH RESEARCH

HIV vaccine research has, to date, excluded children from clinical trials. If researchers were to pursue a clinical trial including children in Kenya, the involvement or exclusion of orphaned or vulnerable children would be critical to the study design. As the HIV epidemic continues in Africa, mortality rates from HIV/AIDS are expected to continue to rise, and the number of children orphaned will continue to increase exponentially (Subbarao \& Coury, 2004). In sub-Saharan Africa, there are an estimated 53 million orphans, with over a quarter orphaned by HIV/AIDS (UNICEF, 2006). Kenya has an estimated population of greater than 39 million people, including an estimated 2.5 million orphans, almost half of whom have been orphaned by the HIV/AIDS epidemic (UNICEF, 2006; Kenya National Bureau of Statistics, 2010). The sizeable population of children orphaned from HIV and other causes argues for the inclusion of this population in biomedical and behavioral research. In addition to the general size of this population, the large number of orphans whose lives have been affected by HIV and who may be at higher risk for HIV positions them as relevant subjects. Guidelines do suggest that individuals, such as wards of the state, identified as vulnerable should not be eligible to participate in research that represents more than greater than minimal risk (US Department of Health and Human Services, n.d.). While it is imperative to ensure the safety of the study population, it is also irresponsible to summarily dismiss a group of individuals without consideration of potential benefit/harm ratios. The definition of minimal risk may vary based on the risks inherent in the group's everyday life. And even the identification of a group as vulnerable or the definition of the term "vulnerable" may require reconsideration. Levine et al. (2004) argue that vulnerability can be both too broadly and too narrowly defined and propose that contextual interpretation of vulnerability should be applied to individuals and groups. Similarly, Shivas (2004) argues that almost all humans can be considered vulnerable in research; however, it is the context of each study by which we should assess potential harm. Analysis protocol by protocol for vulnerability and risk, as opposed to blanket groupings of "vulnerability," ensures that individual and contextual elements are considered. Although minimal risk may be present, it is important to consider the broader context of the procedure to assess for individual vulnerabilities and potential benefits. In order to safely include orphans in research, their unique population vulnerabilities must be directly addressed in a thoughtful and comprehensive manner. Examining three categories of vulnerability-socioeconomic, psychosocial, and medical-experienced by orphaned children in Kenya participating in an HIV 
vaccine trial will provide a lens through which to consider the ethical considerations when involving orphans in biomedical research.

\section{Case Study}

We will consider an HIV vaccine trial that would consist of a nonviral vaccination that is given in a two-part series. The trial seeks to enroll Kenyan participants of ages 18 months to 18 years. The study would be conducted in the north Rift Valley and western Kenyan provinces in a large/moderate-sized city with a large hospital and surrounding satellite clinics. Participants will be recruited from schools, health centers, the Moi Teaching and Referral Hospital, and local organizations. A small financial incentive covering the cost of travel to clinic visits will be provided for all participants. Any participant who comes to a recruitment session will also be reimbursed for travel costs (no more than $400 \mathrm{Ksh} / \mathrm{visit}$ ). During the study, participants would be required to provide a list of all members of the household and proof of custody of that child. Orphans are defined as any child under the age of 18 where either parent or both parents are deceased (UNICEF, 2009). Each participant's status as to orphan, double orphan, or custody arrangement would be noted. Prior to enrollment in the study, participants would be invited to discuss the study and consent in a private environment.

Complete physical exam and mental health screening will be conducted upon enrollment in the study. In addition, each participant will have preliminary testing for HIV and will be retested to ensure seronegative status in accordance with ageappropriate guidelines. Participants enrolled in the study will be followed by a local clinic in Kenya for the duration of the vaccine series and for five years after the vaccinations, with six-month testing for HIV infection and three-month followup visits during the intervals between vaccinations to assess the children's general health status. Parents and guardians will be reminded of each visit with cards and phone calls prior to each visit. Two copies of color-blocked cards with medication schedules and visits will be provided to each participant. Case participants will receive the vaccine, and a matched control cohort will be followed prospectively from the same clinic.

\section{Socio-economic Vulnerabilities of Orphaned Children in Health Research}

There are three central socioeconomic vulnerabilities of orphaned children: their baseline socioeconomic status, educational status of caregivers, and particular economic deprivations for subpopulations that vary by location (Table 1).

SOCIO-ECONOMIC STATUS

First, the baseline economic status of the orphan population should be assessed. Most orphans in sub-Saharan Africa live in poorer households than non-orphans, and their care tends to fall on poorer homes headed by the elderly or by women (Case, Paxson, \& Ableidinger, 2002). A lack of material wealth places orphaned children at increased risk of poorer health and well-being, but also at increased risk in terms of participation in biomedical

TABLE 1. Socioeconomic Vulnerabilities of Orphans.

\begin{tabular}{|c|c|c|c|c|}
\hline Issue & Background & $\begin{array}{l}\text { Ethical Concerns for } \\
\text { Research }\end{array}$ & $\begin{array}{l}\text { Kenya-Specific } \\
\text { Issues }\end{array}$ & Potential Intervention \\
\hline $\begin{array}{l}\text { Baseline } \\
\text { Socio- } \\
\text { economic } \\
\text { Status }\end{array}$ & $\begin{array}{l}\text { Orphans live in poorer } \\
\text { households than non- } \\
\text { orphans (REFS) }\end{array}$ & $\begin{array}{l}\text { Even a small amount of } \\
\text { money could be coercive } \\
\text { for families who have little } \\
\text { or nothing }\end{array}$ & $\begin{array}{l}\text { Cultural awareness } \\
\text { of expectations }\end{array}$ & $\begin{array}{l}\text { - Outline clear expectations at be- } \\
\text { ginning of study using handouts, } \\
\text { video, or pictorial guides } \\
\text { - Community assent to proposed } \\
\text { study }\end{array}$ \\
\hline Education & $\begin{array}{l}\text { Orphan caregivers } \\
\text { likely to have lower } \\
\text { levels of education }\end{array}$ & $\begin{array}{l}\text { Lower literacy and numeracy } \\
\text { mean study goals and } \\
\text { procedures may not be } \\
\text { understood }\end{array}$ & $\begin{array}{l}\text { - Multiple local } \\
\text { dialects } \\
\text { - Uneven literacy } \\
\text { rates } \\
\text { - Concept of being } \\
\text { "taken" }\end{array}$ & $\begin{array}{l}\text { - Translators with skills in common } \\
\text { and vernacular languages } \\
\text { - Handouts using pictures, common } \\
\text { and vernacular language, and } \\
\text { simple sentence structure/ } \\
\text { vocabulary }\end{array}$ \\
\hline $\begin{array}{l}\text { Subpopulation } \\
\text { Specifics }\end{array}$ & $\begin{array}{l}\text { Specific subpopula- } \\
\text { tions such as double } \\
\text { orphans are at } \\
\text { uniquely higher risk } \\
\text { for financial strain }\end{array}$ & $\begin{array}{l}\text { How do you identify, protect, } \\
\text { and appropriately include } \\
\text { these populations? }\end{array}$ & $\begin{array}{l}\text { - Property snatching } \\
\text { from women and } \\
\text { children } \\
\text { - Lack of laws and } \\
\text { rights protect- } \\
\text { ing vulnerable } \\
\text { subpopulations }\end{array}$ & $\begin{array}{l}\text { - Awareness of cultural context } \\
\text { of your study, including social } \\
\text { stressors that may not be appar- } \\
\text { ent to outside observers } \\
\text { - Consider qualitative inquiry to fur- } \\
\text { ther understand specific context }\end{array}$ \\
\hline
\end{tabular}


research. Their increased poverty may make them more susceptible to pressure to participate in studies because of the incentives or perceived benefits they may receive. Orphaned children, or their caregivers, may feel compelled to participate in research in order for their caregivers to receive even small monetary incentives that are not compelling for families with more resources. Researchers must investigate the baseline status for their population and give special consideration to providing appropriate incentives for study participation without inadvertent economic coercion of orphans or their family units. Community expectations and perceptions are significant in evaluating an approach to study recruitment and participation. If research has been conducted in the community before, preconceived ideas about compensation, care, or level of involvement may already exist. While the lower socioeconomic status might render these families more vulnerable to being influenced by financial incentives, it also creates health vulnerabilities for the orphans. These children's increased health risks are an important reason to include them in biomedical research with the potential to benefit their health, yet they might also be susceptible to participation in research to gain access to healthcare and other services.

\section{Case Ethics}

A potential first strategy for investigators would be to conduct online research using worldwide databases to investigate the economic status of the countries of interest. To contextualize this economic information for a particular setting, the researcher could then have conversations with key community members to verify that the study is appropriate for the given community and that proposed incentives are appropriate in accordance with the local economic climate. Community leaders would be a valuable resource for discussions of possible complications and provide introductions to facilitate a discussion with the whole community. Researchers should consider community discussions as a form of community assent for study participation (Naanyu et al., 2011).

\section{EDUCATION}

Frequently, the caregivers responsible for protecting the interests of orphaned children may themselves have only a rudimentary education, speak primarily in local dialect, or be unable to read and write. Delineation of the scope of the research study can be a frustrating communication challenge. Limited education may make it difficult for participants to understand and weigh the concepts of risks and benefits. Lack of numeracy makes it more difficult to decide whether a risk is significant. Perceived or real increased access to healthcare as part of research participation can often act as an independent benefit in the caretaker or child's rationalization process. In economically limited countries where numerous NGOs and other charitable organizations exist, caretakers may have the expectation that the child will be "taken," and this needs to be clarified in the context of the study. In Kenya, many caregivers may believe that participation with a research study or nonprofit organization means that the child will also be "taken," meaning a charitable organization will house, provide food for, and/or educate orphans, sometimes in conjunction with research studies providing healthcare (Nyambedha, 2008). The possibility of an orphan being taken is often an implied benefit in the mind of the caretaker, and so researchers must carefully delineate the scope of care to be provided before and after study participation (ibid.). A study involving orphaned children in this population should take specific steps to address these expectations, including a special emphasis on describing what type of healthcare will be included and the duration of this healthcare for each patient and parent.

\section{Case Ethics}

Potential strategies to convey information about study procedures, risks, and benefits would include using simplified handouts with a basic timeline for literate guardians and children. For less literate persons, a verbal explanation should be provided, the guardian and child can be asked to repeat back the information to clarify comprehension, and studies could also provide a pictorial schedule of clinic visits, the timeline of the study, and associated benefits such as aid with transportation fees. All case materials should be translated into both the common language and local vernacular, and translators should be available at all clinic visits, consents, and procedures.

\section{SUBPOPULATION ECONOMICS}

Particular attention should be paid to identifying any increased risks for subpopulations within this already vulnerable group. In Kenya, recent surveys demonstrated that total orphans, children who have had both parents die, were more often found in the poorest households, indicating that particular types of orphans may be at increased economic disadvantage and thus increased vulnerability (UNICEF, 2006). Within a particular culture, the rights to monetary inheritance and property inheritance can also alter the child's economic status significantly. For example, in sub-Saharan Africa, there exists a practice of property snatching or stealing 
property rights from female-headed or child-headed households, and this is more likely to affect orphans' households (Wakhweya, 2003; Chipfakacha, 2002). In locations where property snatching is prevalent, women can be forced out of their home by their deceased husband's family. If both parents are dead, the father's family may claim the property and forcibly expel the children, leaving them destitute and on the street. These children in turn have significantly decreased resources, increased familial stress, and are, as a result, increasingly vulnerable to financial pressures or the incentives offered by research groups as part of standard practice (Subbarao \& Coury, 2004).

\section{Case Ethics}

Researchers can address the economic vulnerabilities of orphaned children in multiple ways. First, they need to be aware of the particular cultural context of the study, including social stressors that may not be apparent to outside observers. Qualitative inquiry within the community may be a crucial first step to an understanding of the specific context. By increasing awareness of culturally biased socioeconomic stressors, the research team can guard themselves against unintentional population bias. In areas where problems have been identified, for example in areas where property snatching is a known occurrence, it would be unwise to recruit patients for the vaccine study solely using locally established aid centers, since the majority of displaced persons in this community are women and children. Thus, a thorough understanding of the particular economic vulnerabilities of orphans in a particular setting can guide appropriate, protective research methods. The precautions needed may vary based on the type of research. One could argue that, in a case of a simple survey data collection study, neither the risks nor the incentives would be of great enough import to exclude orphans from the study. However, in the case of an HIV vaccine trial, the risks would likely be greater, and the financial incentives used may induce economically deprived, orphaned children to be overly represented and overly exposed to these risks. Examining a community framework with regard to cultural and legal characteristics can provide insight into local economic policy that influences the study population.

\section{Psychosocial Vulnerabilities of Orphaned Children in Health Research}

An orphan's economic vulnerability is significantly related to the psychosocial construct of daily life. Psychosocial vulnerabilities of orphans can be classified into three main areas of focus: caregiver instability, accessibility, and stigma (Table 2).

\section{CAREGIVER INSTABILITY}

Orphanhood shapes the cultural structure around the child in key ways, most notably in terms of which adults are present in the lives of the child, the socioeconomic ramifications of altered family structure, and how the child receives care. Orphans frequently have to move following the death of one or both parents, creating an unstable living environment as they change caregivers (Young \& Ansell, 2003).

The social ramifications from altered family structure, unstable living arrangements, and uncertain social

TABLE 2. Psychosocial Vulnerabilities of Orphans.

\begin{tabular}{|c|c|c|c|c|}
\hline Issue & Background & Relevance & Kenya-Specific Issues & Potential Intervention \\
\hline $\begin{array}{l}\text { Caregiver } \\
\text { Instability }\end{array}$ & $\begin{array}{l}\text { Orphans frequently } \\
\text { have unstable living } \\
\text { arrangements and } \\
\text { history of multiple } \\
\text { caregivers }\end{array}$ & $\begin{array}{l}\text { Unstable living arrange- } \\
\text { ments create tense and } \\
\text { uncertain social frame- } \\
\text { work leading to increased } \\
\text { vulnerability }\end{array}$ & $\begin{array}{l}\text { - Grandparents are frequent } \\
\text { primary caregivers } \\
\text { - Sex of orphan frequently } \\
\text { determines family place- } \\
\text { ment and involvement }\end{array}$ & $\begin{array}{l}\text { Community healthcare work- } \\
\text { ers can provide insight into } \\
\text { local social constructs }\end{array}$ \\
\hline Accessibility & $\begin{array}{l}\text { Orphans frequently } \\
\text { have increased } \\
\text { home workload and } \\
\text { decreased school } \\
\text { attendance }\end{array}$ & $\begin{array}{l}\text { Sample populations often } \\
\text { drawn from school-aged } \\
\text { children }\end{array}$ & $\begin{array}{l}\text { Lack of populations such } \\
\text { as street kids can create } \\
\text { sample bias if drawn from } \\
\text { local schools or daycares }\end{array}$ & $\begin{array}{l}\text { - Diversity in population } \\
\text { recruitment can help } \\
\text { temporize some bias } \\
\text { - Cautious approach to } \\
\text { assent with awareness } \\
\text { of possible educational } \\
\text { deficiencies }\end{array}$ \\
\hline Stigma & $\begin{array}{l}\text { Specific subpopulations } \\
\text { are at uniquely higher } \\
\text { risk }\end{array}$ & $\begin{array}{l}\text { How do you identify, pro- } \\
\text { tect, and appropriately } \\
\text { include these populations? }\end{array}$ & $\begin{array}{l}\text { Children orphaned from HIV } \\
\text { have unique stigmatization } \\
\text { and social isolation }\end{array}$ & $\begin{array}{l}\text { - Awareness of bias in } \\
\text { existing communities } \\
\text { - Extra precautions in } \\
\text { confidentiality } \\
\text { - Screening for mental } \\
\text { health issues }\end{array}$ \\
\hline
\end{tabular}


frameworks increase orphans' vulnerability. As caregivers change, the child must adjust to a new home, family, rules, and expectations (ibid.). The cultural heritage of the child may play a significant role in determining if maternal or paternal relatives gain custody of the child. Orphans may have a tenuous place within a household where their relatives are already under significant financial or social strains. Orphans also may feel more strongly the need to obey the authority figure in charge of their living situation, such as the director of a children's home, out of fear and uncertainty of possible displacement, or because it is cultural to obey those in authority. Orphans may have few if any options to sustain a living if they are kicked out of their adoptive home. These pressures could make a child unlikely to voice any disagreement with a guardian, even if the guardian's decisions place them at risk or discomfort. All these sources of stress within a shifting societal framework can create a situation where children or the families may seek out alternatives means of supporting the family that would not have been previously considered. This could create a pressure to participate in research, discounting potential risks and seeking perceived benefits.

\section{Case Ethics}

Research across 26 sub-Saharan countries, including Kenya, found that care for double orphans varies by gender, with boys more likely to be cared for by grandparents and girls more likely to live with other relatives (UNICEF, 2006). In Kenya, $51 \%$ of double orphans and single orphans not living with a surviving parent are being raised by grandparents (ibid.). The role of grandparents, extended family, or institutions in which orphans may live can have a significant effect on children's ability, willingness, and desire to participate in current research. Having more elderly or female caretakers not only means that orphans live in households with fewer financial resources, but they also may be households that lack status within the community (Case, Paxson, \& Ableidinger, 2002). The combined financial impoverishment and lack of social standing may make orphan caretakers particularly prone to involving their young charges in more risky research if either financial gain or healthcare options are seen as benefits or if involvement is perceived to increase the chance of the child being provided for in other ways. Researchers should be aware of potential complications in custodial arrangements and should require proof of guardianship.

\section{ACCESSIBILITY}

Orphans in sub-Saharan Africa bear an increased workload within both child-headed households and foster families (Subbarao \& Coury, 2004). The increased workload borne by orphans could alter their participation in health research in several ways. Orphans may feel increased responsibility to provide for themselves or their family, and this could exclude them from research participation because they need to work. An increased sense of responsibility to provide for themselves and their family also may increase the risk of influence by potential monetary benefits. Alternatively, as the orphan is required to shoulder a larger portion of the family's responsibilities, caregivers may be less inclined to allow him or her to participate in a study that would remove the child from the workplace for significant amounts of time. There may also be a social desirability bias whereby guardians may not want a child to participate, or be known to researchers, if they are concerned they will get in trouble for not taking care of the child or be perceived to not be providing adequate care.

As increased workload can lead to exclusion from studies due to workforce demands, increased workload can also decrease school participation, decreasing orphaned children's exposure to studies and decreasing the likelihood of literacy and educational development necessary to understand and participate in a study. Lack of participation in school places these children at risk for exclusion from studies through simple geography. The easiest way to survey a population of children and have a consistent way of finding the enrolled children for follow-up or further treatment is through school enrollment. Orphans are at risk of exclusion from such studies as they have higher rates of absence from school and lower enrollment (UNICEF, 2006). This is consistent with studies which have demonstrated that a parent's financial situation is directly related to children's educational outcomes, and as previously discussed, orphans tend to live in poorer households (Ntanda et al., 2009). Studies that solely recruit from schools in a population with a significant number of orphans would have a considerable bias toward underrepresentation of orphans. These selection biases might threaten the scientific validity of a study if the sickest, poorest, or most at-risk subpopulation of children are not evaluated along with others of their age.

In addition to this selection bias, the orphans are also vulnerable due to altered educational experience and health literacy. For example, investigators may need to evaluate the educational resources available and utilized when considering a child's ability to assent. The American Academy of Pediatrics guidelines (1995) state that seven years is the age at which assent can be obtained, based on developmental milestones in children. Children who are socially and educationally deprived may reach these milestones much later in life. 
Children who are not in enrolled in school will fall behind their peers in their ability to read and write. If orphans are to be included in a research study, not only must there be consideration for assent procedures with regard to educational and developmental level, but lack of participation in schooling must be acknowledged as a potential selection bias. In addition, the culture of children not questioning decisions made by the guardians may influence the ability of a child to assent.

\section{Case Ethics}

In our HIV vaccine trial, for example, frequent laboratory monitoring would be required and may be inconvenient for a family who relies on the orphan's work. Attempting to work with families to arrange for convenient testing sites within local communities and flexible hours of clinic offices based on community needs and work schedules may help to improve this outcome. An agricultural-based community, for example, would likely have a better response rate if clinic hours were held after prime working hours or if the vaccination schedule could be targeted to fall outside of peak harvest times.

While alternative methods for explaining projects and signing documents can be designed, it is important to consider the educational limitations of the study protocol when including orphans. The explanation of a complicated scientific concept through pictures or movies may be done, as in the case of an HIV vaccine, but it requires extra investment from the research team. If orphans are to be included in a research study, not only must there be consideration for assent procedures with regard to educational and developmental level, but lack of participation in schooling must be acknowledged as a potential selection bias with orphans underrepresented in a sample drawn from schools.

\section{STIGMA}

Orphans' exposure to abuse, increased stress, and stigma attached to certain subpopulations of orphans are additional psychosocial vulnerabilities that must be considered for ethical health research participation. The increased isolation that accompanies orphans' shifting social framework and stigmatization can lead to increased risk of exploitation and abuse. Studies from Malawi indicate an increased risk of abuse with orphanhood (Muhwezi, Muhangi, \& Mugumya, 2009). Certain orphan populations may face additional burdens. In South Africa and Namibia, orphans who live on the streets have increased exposure to prostitution, drug abuse, HIV, and crime, compounding their traumatic experiences (American Academy of Pediatrics, 1995; Subbarao \& Coury, 2004).
While the subpopulation of street children in research requires additional considerations for research inclusions that are beyond the scope of this paper, exposure to highrisk activities and settings are significant vulnerabilities in orphans. While these increased risks contribute to orphans' vulnerabilities, they also provide an argument for their inclusion in health research. Orphans' increased risk for contracting HIV is precisely why orphans should be included in research related to HIV, particularly research related to vaccine prevention strategies from which this population could greatly benefit. While the risks of vaccine trial participation are typically considered greater than minimal risks, the risk for HIV infection in orphans' daily life is significantly higher. Thus, the argument could be made that adolescents approaching majority age should be able to assent to participation in such a study.

The psychological status of orphans, and the associated vulnerabilities, are also influenced by how they became orphans. In Kenya, large numbers of children are orphaned by AIDS every year. Children orphaned by AIDS may be singled out at school, experiencing stigma and bullying more than other orphans and non-orphans, both of which directly correlate with their mental health (Subbarao \& Coury, 2004; Boris et al., 2006). Children orphaned by AIDS are also more likely to suffer from post-traumatic stress disorder, recurrent trauma, anxiety, and depression, to perform more poorly in school, and to lack self-esteem compared to other orphans (Pelton \& Forehand, 2005; Bhargava, 2005). Studies from Rwanda and Mozambique have cited orphanhood as an independent risk factor for increased depression and social isolation (Boris et al., 2006). Thus, the psychological well-being of the child and the mental health of the population involved should be closely examined and plans enacted to either exclude at-risk participants or provide support to ensure that no further harm is caused. Support provided may include psychological counseling, social services support, or care extended to psychiatric medicine. Studies from Zimbabwe have associated orphanhood with increased stress directly related to decreased social support (Gilborn et al., 2006).

The stigma associated with HIV/AIDS is significant, which also impacts how recruiting, confidentiality, and maintenance of records within the study are handled. Even for a prevention trial for a vaccine, it is critical to consider any possible social ramifications for these children in being associated with such a controversial disease. The stigma that could be associated with participating in an HIV-related trial requires extra protections for their confidentiality. In a population with an already elevated risk of isolation and accompanying mental health issues, the stigma attached to being associated with an HIV trial could be significant and detrimental. 


\section{Case Ethics}

As a researcher, it is crucial to be aware of such comorbidities in a study population, as they render the children more vulnerable. To adhere to the principle of nonmaleficence, a researcher needs to be aware of how orphaned subjects might be at more risk from psychological trauma or from being further stigmatized. Depression scales or other mental health screening tools may be of great use to researchers to identify orphans who may be particularly vulnerable and subsequently provide additional support, such as additional counseling or community resources, for participants. In considering the orphan population for inclusion in a vaccine trial, for example, depression or social isolation might make orphaned children more willing to discount risks to themselves or to overvalue a perceived social benefit from study participation.

\section{Physical Vulnerabilities of Orphaned Children in Health Research}

Physical health, as well as mental health, plays a significant role in the vulnerabilities of orphans related to their developmental stage, differential access to healthcare, and adherence to healthcare routines (Table 3 ).

\section{DEVELOPMENT}

Children are rendered vulnerable because of their developmental process. Because of their developmental immaturity, in most settings, young children cannot provide legally authorized informed consent for their own treatment or for participation in research. Moreover, children lack resources such as money or property that would give them more socioeconomic power. Medically, children have unique parameters for physiologic and psychological development that change constantly with age and growth, creating an additional set of vulnerabilities. Infants can be at greater risk of harm from particular medications or treatments due to physiologic immaturity in how they metabolize medicines or respond to a given therapy, altering the risks and benefits balance they would experience in clinical research. School-age children begin to develop a sense of the permanence of death, influencing their ability to weigh the risks and benefits of a particular course of action. Adolescents are transitioning to physical maturity while still displaying a risk-taking profile that varies significantly from adults. While these are not unique vulnerabilities of orphans, the real versus presumed developmental stage of each orphan must be taken into consideration. Physiologically, nutritionally deprived bodies will not metabolize medications or tolerate treatments in the same manner as healthy organ systems. Thus, evaluation of children for both psychological and physiological development is crucial prior to initiation of a study.

\section{DIAGNOSIS AND TREATMENT}

Orphans may be less likely to access healthcare services. The orphans' role in the family and relationship with their caregiver have been associated with differential presentation to health clinics, government-run institutions, and traditional healers. Orphanhood in Uganda and Kenya has been shown to predict delayed access to healthcare, with orphans with HIV receiving antiretroviral therapy at an older age and, in Ugandan studies, with more advanced disease (Ntanda et al., 2009; Nyandiko,

TABLE 3. Medical Vulnerabilities of Orphans.

\begin{tabular}{|c|c|c|c|c|}
\hline Issue & Background & Relevance & Kenya-Specific Issues & Potential Intervention \\
\hline Presentation of IIIness & $\begin{array}{l}\text { Orphans frequently } \\
\text { present later in } \\
\text { disease process for } \\
\text { medical treatment }\end{array}$ & $\begin{array}{l}\text { Later presentation } \\
\text { can create medical } \\
\text { dilemmas, possibly } \\
\text { excluding orphans } \\
\text { from research }\end{array}$ & $\begin{array}{l}\text { Children presenting } \\
\text { later to HIV clinics for } \\
\text { treatment have more } \\
\text { complicating opportu- } \\
\text { nistic infections }\end{array}$ & $\begin{array}{l}\text { Clear guidelines for } \\
\text { admittance to studies } \\
\text { allowing for varying } \\
\text { presentations at time } \\
\text { of enrollment }\end{array}$ \\
\hline Treatment Facility & $\begin{array}{l}\text { Orphans present } \\
\text { at local clinics, } \\
\text { whereas non- } \\
\text { orphaned children } \\
\text { have a wider range } \\
\text { of presenting } \\
\text { locations }\end{array}$ & $\begin{array}{l}\text { Surveying only large } \\
\text { tertiary care centers } \\
\text { creates population } \\
\text { bias }\end{array}$ & $\begin{array}{l}\text { Systems such as } \\
\text { AMPATH in west- } \\
\text { ern Kenya allow for } \\
\text { greater access to a } \\
\text { varied population with } \\
\text { both urban and rural } \\
\text { clinics }\end{array}$ & $\begin{array}{l}\text { Diversity in sampling } \\
\text { practices through } \\
\text { site variance }\end{array}$ \\
\hline Follow-up/ Supervision & $\begin{array}{l}\text { Unstable social } \\
\text { constructs create } \\
\text { barriers to effec- } \\
\text { tive follow-up and } \\
\text { supervision }\end{array}$ & $\begin{array}{l}\text { Being an orphan has } \\
\text { been associated with } \\
\text { worse medication } \\
\text { adherence but better } \\
\text { clinic attendance in } \\
\text { children infected with } \\
\text { HIV }\end{array}$ & $\begin{array}{l}\text { Adherence trials done } \\
\text { with HIV medications } \\
\text { demonstrate that } \\
\text { social-cultural net- } \\
\text { work is imperative to } \\
\text { assuring adherence }\end{array}$ & $\begin{array}{l}\text { Assessing available } \\
\text { caregivers and } \\
\text { enhancing support } \\
\text { service for retention } \\
\text { in care and research }\end{array}$ \\
\hline
\end{tabular}


Ayaya, \& Nabakwe, 2006). In addition, Deininger et al. (2003) have shown that children in foster care have significantly less access to healthcare services, and a significant increase in the number of days of being sick prior to seeking treatment for orphans. Delays in seeking healthcare also have a significant effect on the health status of children presenting for research studies. Sicker children and their families may be more likely to agree to participation in research out of desperation, but they also may be more vulnerable to any health risks from research participation.

\section{Case Ethics}

Clear inclusion and exclusion criteria for the proposed study are vital, as well as to consider recruiting strategies that will reach children who may not have regular access to the healthcare system. In addition, it would be beneficial to outline how the study will address the varying presentations of orphans at the time of enrollment, as well as plans to meet the health needs of any study participants. In the HIV vaccine trial, it would be important to consider the time delay with seroconversion post-exposure and how to minimize delays in diagnosis among children who might be less likely to return to the clinic.

\section{ADHERENCE}

Not only are orphans sicker at presentation, but issues of adherence to treatment can become a significant issue without parental involvement. Relationship dynamics play a crucial role in adherence to treatment protocols and follow-up healthcare visits. In other work by Vreeman et al. (2009), the presence of "an enabling cultural-social fabric" was the key to the care of children, including pediatric medication adherence, in a setting such as Kenya. The caregiving structures for orphans may play very distinct roles for issues such as taking medicines or bringing a child to clinic; in previous work in Kenya, being an orphan was associated with worse medication adherence among HIV-infected children, but with more consistent clinic attendance (Vreeman et al., 2008). Considering an intervention with long-term outcomes, such as an HIV vaccine trial, the ability to continue to participate in follow-up care and continued health monitoring should be carefully considered.

\section{Case Ethics}

The social support that orphans do or do not receive over time can have a critical impact on their health and well-being when participating in biomedical research. Providing these children with additional resources such as transportation reimbursement for clinic visits may help to improve clinic visit show rates. Concordantly, medication adherence could be increased by simplifying dosing regimens and using packaging appropriate for children and people who may be illiterate.

\section{Conclusion}

Pediatric orphans are a vulnerable population due to decreased economic resources, psychosocial instability, increased risk of abuse, and delayed/decreased access to healthcare. These vulnerabilities are significant, and they have the potential to affect the orphans' ability, willingness, and likelihood to participate in biomedical research. For researchers, these vulnerabilities are an additional set of concerns that must be addressed in a populationspecific manner to ethically include or exclude participation. Precautions need to be taken to protect orphans and to address these vulnerabilities when developing protocols for health research in settings with large orphan populations. By carefully considering each issue in a population- and study-specific manner, researchers can make valuable contributions to the overall health and well-being of this uniquely vulnerable population.

\section{Acknowledgments}

This project was supported in part by an award to Dr. Braitstein from the Eunice Kennedy Shriver National Institute of Child Health \& Human Development (R01HD060478), by an award to Dr. Vreeman from the National Institute for Mental Health (1K23MH087225), and by a grant to the Academic Model Providing Access to Healthcare (AMPATH) Partnership from the United States Agency for International Development. The content is solely the responsibility of the authors and does not necessarily represent the official views of any of these institutions.

\section{Author Note}

Address correspondence to: Rachel T. Thompson, Fellow, Children's Health Services Research, Indiana University School of Medicine, HITS Building, Room 1020, 410 West 10th Street, Indianapolis, IN 46202. Phone: 317-278-0552; Fax: 317-278-0456; E-MAIL: rtthomps@iupui.edu.

\section{Authors' Biographical Sketches}

Rachel Thompson is a general pediatrician and fellow in Children's Health Services Research at Indiana University School of Medicine. She is actively pursuing 
a career in health services research and has an interest in international research. She led the conception, design, drafting, and critical revision of the manuscript.

Eric Meslin is Director of the Indiana University Center for Bioethics; Associate Dean for Bioethics in the Indiana University School of Medicine; and Professor of Medicine, Medical and Molecular Genetics, Public Health and Philosophy. He co-directs the IU Center for Law, Ethics and Applied Research in Health Information (CLEAR), and the IU-Moi Academic Research Ethics Partnership, an innovative bioethics research training program in Eldoret, Kenya. He participated extensively in the development of this analysis, with significant contributions to the conception, design, writing, and revisions of the final manuscript.

Paula Braitstein is Associate Research Professor of Medicine at Indiana University School of Medicine, Co-Field Director for Research for AMPATH, and Co-Principal Investigator for the OSCAR research study in western Kenya. Her research expertise lies in evaluating care outcomes including for HIV-infected patients, orphans, separated children, and other vulnerable populations. She contributed to the data interpretation and to critical revision of the manuscript.

Winstone Nyandiko is Associate Professor and the Head of the Department of Child Health and Paediatrics, School of Medicine, Moi University College of Health
Sciences in Eldoret, Kenya, and Associate Program Manager for the AMPATH partnership. His expertise lies in evaluating the clinical management of HIVexposed and HIV-infected children and implementation research in Kenya. He contributed to the data interpretation and critical revision of the manuscript.

Samuel Ayaya is Professor in the Department of Child Health and Paediatrics, School of Medicine, Moi University College of Health Sciences in Eldoret, Kenya, and Co-Chair of the Kenya Pediatric Research Working Group. His research interests include children's nutrition, growth, and development, the progression of HIV in children, and child abuse and neglect. He contributed to the data interpretation and critical revision of the manuscript.

Rachel Vreeman is Assistant Professor of Pediatrics at Indiana University School of Medicine and Co-Director for Pediatric Research for the Indiana University-Kenya Partnership and Academic Model Providing Access to Healthcare (AMPATH). She has extensive research experience in western Kenya that focuses on improving the provision of healthcare to children in resource-limited settings, with particular expertise in children's adherence to HIV treatment. She participated extensively in the development of this analysis, with significant contributions to the conception, design, drafting, and critical revisions of the final manuscript.

\section{References}

American Academy of Pediatrics. (1995). Guidelines for the ethical conduct of studies to evaluate drugs in pediatric populations (Committee on Drugs, American Academy of Pediatrics). Pediatrics, 95(2), 286-294.

Beauchamp, J. (2009). Principles of biomedical ethics. New York: Oxford University Press.

Bhargava, A. (2005). AIDS epidemic and the psychological well-being and school participation of Ethiopian orphans. Psychology, Health, and Medicine, 10(3), 263-275.

Boris, N., Thurman, T., Snider, L., Spencer, E., \& Brown, L. (2006). Infants and young children living in youth-headed households in Rwanda: Implications of emerging data. Infant Mental Health Journal, 27(6), 584-602.

Case, A., Paxson, C., \& Ableidinger, J. (2002). Orphans in Africa: Center for Health and Well-being, Research Program in Development Studies. Princeton, NJ: Princeton University.

CHIPfakacha, V. (2002). Comparison of orphan coping mechanisms in the old (pre-colonial) and new (post-colonial) Shona (Zimbabwe), Ndebele (Zimbabwe, South Africa), Kalanga (Botswana, Zimbabwe), and Tswana (Botswana, South Africa) cultures. Botswana: World Health Organization.
Deininger, K., Garcia, M., \& Subbarao, K. (2003). AIDS-induced orphans as systemic shock: Magnitude, impact and program interventions in Africa. World Development, 31(7), 1201-1220.

Gilborn, L., Apicella, L., Brakarsh, J., Dube, L., Jemison, K., Kluckow, M., Smith, T., \& SNider, L. (2006). Orphans and vulnerable youth in Bulawayo, Zimbabwe: An exploratory study of psychological well-being and psychosocial support programs. New York: Population Council.

Kenya National Bureau of Statistics. (2010). Kenya 2009 population and housing census highlights. Retrieved from http://www. knbs.or.ke/Census\%20Results/KNBS\%20Brochure.pdf.

KIPNIS, K. (2003). Seven vulnerabilities in the pediatric research subject. Theoretical Medicine and Bioethics, 24(2), 107-120.

Levine, C., Faden, R., Grady, C., Hammerschmidt, D., Eckenwiler, L., Sugarman, J., Et AL. (2004). The limitations of "vulnerability" as a protection for human research participants. American Journal of Bioethics, 4(3), 44-49.

Muhwezi, W., Muhangi, D., \& Mugumya, F. (2009). Intrahousehold differences in health seeking behavior for orphans and non-orphans in an NGO-supported and non-supported subcounty of Luwero, Uganda. African Health Sciences, 9(2), 109-117. 
NaAnyu, V., Sidle, J., Frankel, R. M., Ayuku, D., Nyandiko, W. M., \& INUI, T. S. (2011). Rooting inquiry in tradition: The health baraza as a tool for social research in Kenya. Qualitative Health Research, 21(1), 14-26.

Ntanda, H., Olupot-Olupot, P., Mugyenyi, P., Kityo, C., Lowes, R., Cooper, C. ET AL. (2009). Orphanhood predicts delayed access to care in Ugandan children. Pediatric Infectious Disease Journal, 28(2), 153-155.

Nyambedha, E. (2008). Ethical dilemmas of social science research on AIDS and orphanhood in Western Kenya. Social Science and Medicine, 67(5), 771-779.

Nyandiko, W., Ayaya, S., \& Nabakwe, E. (2006). Outcomes of HIV-infected orphaned and non-orphaned children on antiretroviral therapy in Western Kenya. Journal of Acquired Immune Deficiency Syndrome, 43(4), 418-425.

Pelton, J. \& Forehand, R. (2005). Orphans of the AIDS epidemic: An examination of clinical level problems of children. Journal of the American Academy of Child and Adolescent Psychiatry, 44(6), 585-591.

Shivas, T. (2004). Contextualizing the vulnerability standard. American Journal of Bioethics, 4(3), 84-86.

Subbarao, K., \& Coury, D. (2004). Reaching out to Africa's orphans: A framework for public action. Washington, DC: World Bank Publications.

UNICEF. (2006). UNICEF Africa's orphaned and vulnerable generations: Children affected by HIV/AIDS. Retrieved from
http://www.unicef.org/publications/files/Africas_Orphaned_ Generation_Executive_Summary_Eng.pdf.

UNICEF. (2009). Orphans. Retrieved from http://www.unicef. org/media/media45279.html.

US Department of Health and Human Services. (1979). The Belmont Report. Retrieved from http://www.hhs.gov/ohrp/ humansubjects/guidance/belmont.html.

US Department of Health and Human Services. (N.D.). US Department of Health and Human Services Code of Federal Regulations: Protection of human subjects. Retrieved from http://www.hhs.gov/ohrp/humansubjects/ guidance/45cfr $46 . \mathrm{html}$.

Vreeman, R., Nyandiko, W. A., Walumbe, E., Marrero, D., \& INUI, T. S. (2009). Factors sustaining pediatric adherence to antiretroviral therapy in western Kenya. Qualitative Health Research, 19(12), 1716-1729.

Vreeman, R., Wiehe, S., Ayaya, S., Musick, B., \& Nyandiko, W. (2008). Association of antiretroviral and clinic adherence with orphan status among HIV-infected children in western Kenya. Journal of Acquired Immune Deficiency Syndromes, 49(2), 163-170.

WaKhWEya, A. (2003). Health strategies to mitigate the impact of orphanhood. Presentation at the OVC Workshop, World Bank, Washington, DC.

Young, L. \& Ansell, N. (2003). Young AIDS migrants in South Africa: Policy implications for empowering children. AIDS Care, 15(3), 337-345. 
Reproduced with permission of the copyright owner. Further reproduction prohibited without permission. 